\title{
Together apart
}

\begin{abstract}
A year on from the last-minute cancellation of the 2020 American Physical Society March Meeting, we examine the ups and downs of the online conference experience that has become the new normal during the COVID-19 pandemic.
\end{abstract}

n early March 2020, thousands of physicists around the world were packing bags, travelling to airports and sitting on planes bound for Denver, or already checking into hotels, when they heard the news: the American Physical Society had cancelled the March Meeting due to the unfolding COVID-19 pandemic. It seemed a dramatic move at the time, but it was ultimately the right call. The Division of Soft Matter was the first to take its sessions online $^{1}$ and others soon followed suit.

Since then, almost all conferences - big and small - have become online-only events, along with colloquia, $\mathrm{PhD}$ defences, grant panels and, in many places, lectures and seminars. This year's March Meeting is no exception. One year into this online-only experiment is a natural point to reflect on the distanced exchange of ideas and how it has served the scientific community.

For centuries, scientists have gathered to present and discuss their work. When conferences switched to an online format in 2020, many focussed on ensuring that researchers could still disseminate their work, relying on webinar platforms to do so. The question-and-answer session is usually moved to the chat function, which has some advantages. The conference chair can moderate the questions, and the written format can help to mediate language barriers and hearing difficulties.

But, of course, even at face-to-face meetings, meaningful discussions rarely happen during the few minutes directly after a talk but mostly during the breaks. Many fruitful ideas and many productive collaborations have been hatched over a cup of coffee between sessions. In real life, this kind of encounter is often unplanned, but serendipity is not a native feature of online communication.

Limited by existing conferencing software, many large meetings did away with coffee breaks altogether, whereas some smaller conferences took them online. Virtual breakout rooms can - to some extent simulate the queue for the refreshments table and give attendees the opportunity to strike up a conversation. Some, like the Photonics Online Meetup - online since its inception - have experimented with virtual reality poster sessions ${ }^{2}$ (pictured) and breaks to foster more informal interactions.

Beyond facilitating scientific discussions around a specific talk or poster, conferences

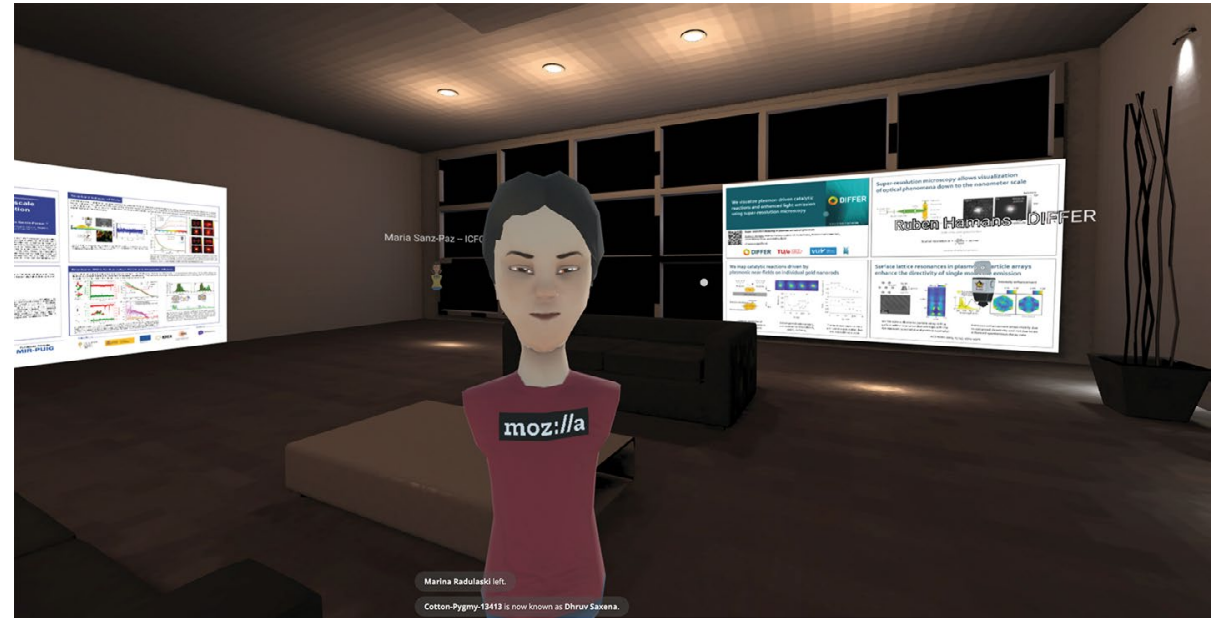

Credit: Riccardo Sapienza

and workshops play an important role in building a strong research community and establishing a common culture. The Gordon Research Conference series is renowned for its commitment to nurturing research communities, and the organizers decided to defer their recent events rather than move them online. The reason? A dearth of platforms that could faithfully recreate the environment their meetings offer (https:// www.glycobiology.org).

However, online conferences could play their part in making research communities more equitable. Holding meetings in virtual spaces rather than at swanky conference venues levels the playing field for less well-off institutions. By eliminating the need to travel, online conferences and seminars reach larger audiences and can draw on a wider pool of speakers than their traditional counterparts. The virtual format also makes conferences more accessible ${ }^{3}$ for disabled researchers.

But there is a flip side. Fully participating in and benefiting from an online conference requires an unimpeded broadband Internet connection - far from a given in large parts of the world ${ }^{4}$. This digital divide is only exacerbated by the use of virtual-reality elements. Although an online format may alleviate some social pressures, attending a conference in a different time zone, often while also working full time, does not necessarily benefit researchers with familial responsibilities.
Although online conferences have kept scientific exchange going during the COVID-19 pandemic, and their contribution to reducing researchers' carbon footprint ${ }^{5}$ is clear, they are not a panacea. But they are here to stay, even once face-to-face meetings are deemed safe again. A new conference calendar may evolve with a mix of in-person, online-only and hybrid events.

Without the pressure to retrofit what was originally planned as a face-to-face meeting, organizers must take care to not reduce online conferences to a means for disseminating content alone. Developing the platforms to replicate the buzz of a large meeting or the community feel of a topical workshop without excluding swathes of the global research community may seem like a daunting task, but it will be worth the effort. Ultimately, meeting people to exchange ideas is the reason why researchers travel rather than stay in their office, reading the conference proceedings.

Published online: 9 March 2021 https://doi.org/10.1038/s41567-021-01209-z

\footnotetext{
References

1. Middleton, C. Phys. Today https://doi.org/fw36 (2020).

2. Virtual reality poster session. Photonics Online Meetup http:// go.nature.com $/ 3 \mathrm{sr} 6 \mathrm{jOz}(2020)$.

3. Vasquez, K. Sci. Am. http://go.nature.com/2NDv7nP (2021).

4. Worldwide broadband speed league 2020. Cable.co.uk http:// go.nature.com/3dHWyra (2020).

5. Burtscher, L. et al. Nat. Astron. 4, 823-825 (2020).
} 